\title{
A Complex, Multidisciplinary Approach to Prevention of Gastro-Duodenal Bleeding in Therapeutic Patients of a General Hospital
}

\author{
Andrey V. Budnevsky, $\mathrm{PhD}, \mathrm{ScD}^{1}$; Evgeniy F. Cherednikov, $\mathrm{PhD}, \mathrm{ScD}^{1}$; \\ Artyom V. Popov ${ }^{1}$; Evgeniy S. Ovsyannikov, $\mathrm{PhD}^{1 *}$; Andrey Y. Kravchenko, PhD, ScD ${ }^{1}$; \\ Andrey Y. Kuranosov, $\mathrm{PhD}^{2}$; Konstantin O. Fursov² \\ ${ }^{1}$ Voronezh State Medical University named after N. N. Burdenko \\ ${ }^{2}$ Voronezh City Emergency Care Hospital \\ Voronezh, the Russian Federation
}

\begin{abstract}
The aim of this study was to make the prevention of erosive-ulcerous gastroduodenal bleeding (EU-GDB) more efficient in therapeutic patients admitted to a general hospital by using a multidisciplinary approach that includes a diagnostic algorithm, treatment protocol, and individual methods of EU-GDB prevention.

Materials and Methods: The study included 114 patients of the therapeutic, pulmonary, and cardiology departments of the Voronezh City Emergency Care Hospital. The patients had been admitted due to destabilization of their underlying conditions and displayed signs of acute gastroduodenal erosions and ulcers during their stay in the hospital. All the patients were randomly divided into two equal groups: the main group and the comparison group. A multidisciplinary approach was applied to patients of the main group ( $\mathrm{n}=58$; mean age, $62.64 \pm 14.37$ ); it included early pre-clinical diagnosis of erosive-ulcerous gastroduodenal impairments (EU-GDI) by fibrogastroduodenoscopy on the second or third day after their admission to the hospital, which helped to reveal in a timely manner uncomplicated EU-GDI and directly start local treatment as a part of complex therapy. An algorithm of the procedure provides for participation of a surgeon. Powder-like biologically active granular sorbents of the new generation (ASEPTISORB-A, ASEPTISORB-D, or ASEPTISORB-DT) were applied to the revealed acute erosions and gastroduodenal ulcers during the FGDS procedure to prevent hemorrhagic complications. After manifestation of the first signs of EU-GDB: during the curative endoscopy, the use of the developed minimally invasive method of endoscopic hemostasis, which provided combined application of a local haemostatic preparation Gelplastan and ASEPTISORB-D to the defect area. In the comparison group ( $\mathrm{n}=56$ ), the traditional technique of surgical consultations "on demand" was used. FGDS was performed when first symptoms appeared. Common methods of endoscopic hemostasis without local treatment of EU-GDI and application of granulated sorbents were used in this group.
\end{abstract}

Conclusion: The developed program helps to prevent hemorrhagic complications, exclude emergency operations, and reduce mortality rate by 3 times. (International Journal of Biomedicine. 2017;7(3):204-207.)

Key Words: erosive-ulcerous damages • gastroduodenal bleedings • granular sorbents • endoscopic hemostasis

\section{Introduction}

The problem of gastroduodenal bleeding (GDB) in the context of therapeutic pathology and the cardiovascular system disorders is still one of the most acute problems in clinical medicine. ${ }^{(1-3)}$ Timely diagnosis of symptomatic

*Corresponding author: Evgeniy S. Ovsyannikov, PhD Department of faculty therapy, Voronezh State Medical University named after N.N. Burdenko.Voronezh, Russia. E-mail: ovses@, yandex.ru. gastroduodenal erosions and ulcers is a challenge that still does not have a solution because $30 \%-90 \%$ of patients have an asymptomatic course of these conditions, $46 \%-58 \%$ patients do not display a typical clinical performance, and in $25 \%-42 \%$ of patients, symptoms of the underlying condition prevail. In most cases, acute erosions and ulcers are diagnosed only in cases of bleeding or autopsy. ${ }^{(1,3)}$

Causes of the consistently high level of mortality in therapeutic patients with GDB are reported to be, first, untimely diagnosis of gastroduodenal erosions and ulcers that result in massive bleeding; second, high operational risk in patients 
with decompensation of an underlying chronic condition in the context of anemia; third, a lack of unified and successive tactics of medical care. ${ }^{(1,3)}$ Thus, prevention and treatment of erosive-ulcerous GDB (EU-GDB) in patients with therapeutic pathologies are acute problems in applied medicine.

The aim of this study was to make the prevention of EU-GDB more efficient in therapeutic patients admitted to a general hospital by using a multidisciplinary approach that includes a diagnostic algorithm, treatment protocol, and individual methods of EU-GDB prevention.

\section{Materials and Methods}

The study included 114 patients of the therapeutic, pulmonary, and cardiology departments of the Voronezh City Emergency Care Hospital. The patients had been admitted due to destabilization of their underlying conditions and displayed signs of acute gastroduodenal erosions and ulcers during their stay in the hospital. The investigation was approved by local ethics committee, and written informed consent was obtained from all participants.

Reasons for hospitalization were hypertension and diabetes mellitus (27.2\%); the active stage of COPD, bronchitis and pneumonia (14.9\%); progressive angina pectoris $(10.5 \%)$; and ischemic heart disease and heart rhythm disorders (34.2\%).

Depending on the character of gastroduodenal erosiveulcerous impairments, all patients were divided into several groups. In the first group were patients, 49(43\%), with isolated or multiple erosions in the stomach and/or duodenum; in the second group were patients, 47(41.2\%), with acute gastric and/ or duodenal ulcers. (Unlike chronic gastroduodenal ulcers, acute gastric and/or duodenal ulcers were located on all walls of the organ.); and in the third group were patients, $18(15.8 \%)$, with ulcerous gastric and/or duodenal disease.

Erosive and ulcerous defects in therapeutic patients either showed no signs of bleeding or were the causes of hemorrhages. Among 49 patients with acute gastric and/ or duodenal erosions, $18(15.8 \%)$ displayed signs of erosive bleeding. Among 47 patients with gastroduodenal ulcers, $24(20.1 \%)$ were found to have signs of acute GDB. Bleeding stigmata of peptic ulcers were classified according to the Forrest (F) classification. ${ }^{(4)}$

All the patients were randomly divided into two equal groups: the main group and the comparison group. Patients of the main and comparison groups were comparable in their age, gender, clinical performance, accompanying conditions, localization and size of erosive-ulcerous defects, and duration of observation. Similar pharmacological therapy conforming to the current standards for care of patients with therapeutic pathologies was used in both groups. Patients of both groups received antiulcerous therapy: proton pump inhibitors (omeprazole), antacids, anti-helicobacter therapy (on indications).

A multidisciplinary approach was applied to patients of the main group ( $n=58$; mean age, 62.64 \pm 14.37 ); it included early pre-clinical diagnosis of erosive-ulcerous gastroduodenal impairments (EU-GDI) by fibrogastroduodenoscopy (FGDS) on the second or third day after their admission to the hospital, which helped to reveal in a timely manner uncomplicated EUGDI and directly start local treatment as a part of complex therapy. An algorithm of the procedure provides for participation of a surgeon. Powder-like biologically active granular sorbents of the new generation (ASEPTISORB-A, ASEPTISORB-D, or ASEPTISORB-DT) were applied to the revealed acute erosions and gastroduodenal ulcers during the FGDS procedure to prevent hemorrhagic complications. The mechanism of action of these sorbents is based on a potent draining effect, which results in complete cleaning from the wound of the wound contents: clots of blood, fragments of tissues, microorganisms, and the decay products of these contents. Next, regeneration and epithelialization processes are activated. ASEPTISORB-A contains an anesthetic agent, anilocaine, that removes the pain syndrome. ASEPTISORB-D contains an antiseptic agent, dioxydin, that prevents development of a purulent process. ASEPTISORB-DT contains dioxydin and a protein-degrading enzyme, collagenase, that lyses necrotic tissues.

Patients of the main group with acute erosions underwent local treatment by endoscopic insufflation of ASEPTISORB-A; patients with gastroduodenal ulcers less than $1.0 \mathrm{~cm}$ in diameter were given a local treatment by pneumatic insufflation of a granular sorbent ASEPTISORB-D on the defect area. Patients with ulcerous defects $1.0 \mathrm{~cm}$ and more were given combined insufflations of two sorbents: first, $0.2 \mathrm{~g}$ of ASEPTISORB-DT was applied on the bottom of the ulcer using an insufflator; then, $0.4 \mathrm{~g}$ of ASEPTISORB-D was applied on the whole surface of the ulcer. Second-stage treatment of the ulcerous process (after cleansing the ulcerous bottom of necrotic tissues) was done using insufflations of ASEPTISORB-D only. Local treatment of erosive-ulcerous defects was performed with a 4-5 day interval.

The plans of action were altered after manifestation of the first signs of EU-GDB: during the curative endoscopy, the use of the developed minimally invasive method of endoscopic hemostasis, which provided combined application of a local haemostatic preparation Gelplastan and ASEPTISORB-D to the defect area. A surgeon participating in this procedure helped to choose the further tactics of treatment according to the treatment protocol of patients with GDB.

The comparison group consisted of 56 patients (mean age, 62.38 \pm 14.47 ). In this group, the traditional technique of surgical consultations "on demand" was used. FGDS was performed when first symptoms appeared. Common methods of endoscopic hemostasis without local treatment of EU-GDI and application of granulated sorbents were used in this group.

The statistical analysis was performed using the statistical software STATISTICA 6.0. A probability value of $P<0.05$ was considered statistically significant.

\section{Results and Discussion}

The results obtained demonstrated that clinical and endoscopic remission in patients of the main group came earlier than in patients of the comparison group. A timely diagnostic FGDS performed as a part of multidisciplinary approach gave an opportunity to reveal acute EU-GDI without signs of bleeding (F-III) early in 41 out of 58(70.7\%) patients 
of the main group. To these patients, we applied individual methods of local treatment to prevent GDB with biologically active granulated sorbents according to the technique we developed. It should be noted that granulated sorbents swell directly after insufflations, forming an elastic, hardly detached hydrogel covering on the surface of the erosive-ulcerous defect, protecting it from aggressive gastric and duodenal content. This layer of hydrogel covered a defect as a sort of a curative dressing, under which the reparation process proceeded significantly more rapidly. Endoscopic investigations showed that after local treatment with granulated sorbents, all patients of the main group manifested rapid, high quality healing of erosive-ulcerous defects. Furthermore, special attention was paid to two important moments relating to preventive local treatment by granulated sorbents: first, gastric pain syndrome disappeared in all patients on the first day of treatment, and, second, no one patient of the main group displayed signs of hemorrhagic complications.

Another important peculiarity of the timely FGDS, as a part of multidisciplinary approach, appeared to be detection of acute gastroduodenal erosions and ulcers complicated by bleeding in $17(29.3 \%)$ patients. As the study demonstrated, $12(70.6 \%)$ of these 17 patients were diagnosed with bleeding in the initial stage (F-IIC), 4(23.5\%) patients had bleeding with unstable hemostasis (F-IIA - F-IIB), and only 1(5.9\%) patient was reported to have active bleeding (F-IA - F-IB), which demanded endoscopic arrest of the hemorrhage. All these patients of the main group were given the minimally invasive method of local hemostasis as a part of the multidisciplinary approach to prevent recurrence of bleeding; this method provided combined application of powder-like Gelplastan and ASEPTISORB-D on the defect area during the procedure of curative endoscopy. As observations demonstrated, the applied pharmaceutical complex turned into a blood-painted massive layer of hydrogel directly after pneumo-insufflation and protected a thrombosed vessel, clot or hematin from lysis, preventing repeated hemorrhages in the complex treatment with general hemostatic and antiulcerous therapy. Such a complex program of treatment resulted in a lack of hemorrhage recurrence and emergency operations in patients of the main group. Hospital length of stay (LOS) in the main group was $8.86 \pm 1.69$ days. One $(1.7 \%)$ patient of the main group, who was admitted to the department of general therapy with acute severe cardiovascular and respiratory failure and atrial fibrillation after acute myocardial infarction and an acute cerebrovascular event, died. Uncontrolled intake of warfarin resulted in GDB in this patient. GDB was arrested by the developed endoscopic technique, but the patient died in 3 hours 15 minutes after admission due to underlying cardiovascular and respiratory failure on the background of anemia.

In the patients of the comparison group, pain syndrome related to EU-GDI persisted until they were discharged from the hospital on the eighth to tenth day. During endoscopic investigation, only $32.1 \%$ patients of the comparison group displayed erosive-ulcerous gastroduodenal defects without signs of F-III bleeding. Acute gastroduodenal erosions and ulcers complicated by hemorrhages were diagnosed in the rest $(67.9 \%)$ of the patients in the comparison group. Endoscopic findings of GDB were classified as follows: F-IIC in 22 $(39.3 \%)$ patients, F-IIA and F-IIB in $14(25 \%)$ patients, and F-IA in 2(3.6\%) patients. Hemorrhage recurrences in patients of the comparison group were registered in $6(10.7 \%)$ patients, 2 of them were operated on during the peak of the bleeding rate. In the comparison group, 3(5.4\%) patients died. The hospital LOS in the comparison group was $12.47 \pm 3.44$ days vs. $8.86 \pm 1.69$ days in the main group $(P<0.05)$. Comparative efficiency of EU-GDB prevention in therapeutic patients of a general hospital is presented in Table 1 .

\section{Table 1.}

Efficiency of EU-GDB prevention in patients of the main and comparison groups

\begin{tabular}{|l|c|c|c|}
\hline \multicolumn{1}{|c|}{ Variable } & $\begin{array}{c}\text { Main } \\
\text { group } \\
(\mathrm{n}=58)\end{array}$ & $\begin{array}{c}\text { Comparison } \\
\text { group } \\
(\mathrm{n}=56)\end{array}$ & $P$ \\
\hline $\begin{array}{l}\text { Detection of acute erosions } \\
\text { and ulcers in endoscopic } \\
\text { investigation (day) }\end{array}$ & 2nd - 3rd & 6th -8th & - \\
\hline $\begin{array}{l}\text { Number of patients with acute } \\
\text { erosions and ulcers without } \\
\text { signs of bleeding, (n/\%) }\end{array}$ & $41 / 70.7 \%$ & $18 / 32.1 \%$ & $<0.05$ \\
\hline $\begin{array}{l}\text { Number of patients with } \\
\text { bleeding in the initial stage, } \\
\text { (n/\%) }\end{array}$ & $12 / 20.7 \%$ & $22 / 39.3 \%$ & $<0.05$ \\
\hline $\begin{array}{l}\text { Number of patients with } \\
\text { unstable hemostasis, (n/\%) }\end{array}$ & $4 / 6.9 \%$ & $14 / 25 \%$ & $<0.05$ \\
\hline $\begin{array}{l}\text { Number of patients with active } \\
\text { bleeding, (n/\%) }\end{array}$ & $1 / 1.7 \%$ & $2 / 3.6 \%$ & - \\
\hline Recurrence of bleeding, (n/\%) & - & $6 / 10.7 \%$ & - \\
\hline Surgery, (n/\%) & - & $2 / 3.6 \%$ & - \\
\hline Mortality rate, (n/\%) & $1 / 1.7 \%$ & $3 / 5.4 \%$ & - \\
\hline LOS, (days) & 8.86 & 12.47 & $<0.01$ \\
\hline
\end{tabular}

Thus, timely endoscopic examination of the stomach and duodenum in therapeutic patients helped to increase the detection of acute EU-GDI by 2.2 times before the occurrence of hemorrhagic complications. Early local preventive treatment of EU-GDI according to the technique we designed gave an opportunity to prevent the development of decompensated conditions of the underlying therapeutic pathology and reduce the hospital LOS by 1.4 times. In a meta-analysis, endoscopic management was a more effective treatment than pharmacological managements or placebo for lowering the rebleeding and mortality rates in patients with GDB. ${ }^{(5)}$ Early endoscopic management has been reported to decrease the length of hospitalization, in comparison with delayed endoscopy, in high- and low-risk groups. ${ }^{(6,7)}$

\section{Conclusion}

Thus, the complex curative program we developed to prevent GDB in therapeutic inpatients is based on a 
multidisciplinary approach: an improved algorithm of examination, timely diagnosis of symptomatic EU-GDI, and pharmacological therapy with early local preventive treatment of acute erosions and gastroduodenal ulcers by granulated sorbents. This program helps to prevent hemorrhagic complications, exclude emergency operations, and reduce mortality rate by 3 times.

\section{Competing interests}

The authors declare that they have no competing interests.

\section{References}

1. Kolobov SV, Zayrat'yants OV, Loranskaya ID, Simonova NI, Poputchikova E.A. [Erosive-ulcerative lesions of the upper gastrointestinal tract in patients with acute myocardial infarction]. Neot Ter. 2002;( 3-4): 63 67. [Article in Russian].

2. Cherednikov EF, Kunin AA, Cherednikov EE, Moiseeva NS.
The role of etiopathogenetic aspects in prediction and prevention of discontinuous-hemorrhagic (Mallory-Weiss) syndrome. EPMA J. 2016; 7:7. doi: 10.1186/s13167-016-0056-4.

3. Budnevskiy AV, Popov AV, Cherednikov EF, Kuranosov AY. [Erosive-ulcerous gastroduodenal impairments in therapeutic patients]. Nauchno-meditsinskiy vestnik Tsentral'nogo Chernozem'ya. 2016; 65:63-70. [Article in Russian].

4. Forrest JA, Finlayson ND, Shearman DJ. Endoscopy in gastrointestinal bleeding. Lancet. 1974;2(7877):394-7.

5. Cook DJ, Guyatt GH, Salena BJ, Laine LA. Endoscopic therapy for acute nonvariceal upper gastrointestinal hemorrhage: a meta-analysis. Gastroenterology. 1992;102(1):139-48.

6. Lin HJ, Wang K, Perng CL, Chua RT, Lee FY, Lee $\mathrm{CH}$, Lee SD. Early or delayed endoscopy for patients with peptic ulcer bleeding. A prospective randomized study. J Clin Gastroenterol. 1996;22(4):267-71.

7. Jang JY. Recent Developments in the Endoscopic Treatment of Patients with Peptic Ulcer Bleeding. Clin Endosc. 2016;49(5):417-420. 